\title{
NONLINEAR OSCILLATIONS AND BUCKLING OF ANISOTROPIC CYLINDRICAL SHELLS UNDER LARGE INITIAL STRESSES
}

\author{
RASAJIT KUMAR BERA ${ }^{1}$
}

(Received 11 September 1987; revised 15 September 1988)

\begin{abstract}
The large-amplitude oscillations and buckling of an anisotropic cylindrical shell subjected to the initial inplane biaxial normal stresses have been analysed. The concept of anisotropy used by Lekhnitsky has been introduced into the field equations for cylindrical shells of isotropic material deduced by Donnell. The method of Galerkin and the method of successive approximation have been used to obtain the desired approximate solution. The expression for the critical loads for the buckling of anisotropic cylindrical shells has been obtained during intermediate stages of analysis. Some relevant frequency response graphs of the obtained solution are also presented. The minimum critical loads for various classes of anisotropy have also been given at the end of the discussion, to exhibit the effects of large deflections and imperfections on elastic buckling.
\end{abstract}

\section{Introduction}

In recent years, the parametric response of structures has been reexamined in the new evolved theory of dynamic stability of elastic systems. Several nonlinear problems of the dynamic stability of isotropic plates were investigated by Bolotin [2]. So far as the linear parametric response of isotropic plates is concerned, Evan-Iwanouski [4] cites ten items, one of which, by Ambartsumian and Khachaturian [1], is devoted to small oscillations of anisotropic plates. Nowinski [8] has given a detailed analysis of the nonlinear oscillation of anisotropic plates.

\footnotetext{
${ }^{1}$ Department of Mathematics, Presidency College, Calcutta, 700073, West Bengal, India.

(C) Copyright Australian Mathematical Society 1990, Serial-fee code 0334-2700/90
} 
The present paper is concerned with the analysis of large-amplitude oscillations of an anisotropic cylindrical shell subjected to the initial inplane biaxial normal stresses. Following the analysis of Donnell [3] for cylindrical shells of isotropic material, the field equations of anisotropic cylindrical shells have been derived. The concept of anisotropy is the same as that given by Lekhnitsky [6]. The deflection of the shell has been represented in a simple separable form, satisfying the conditions on the boundaries, and a particular integral has been obtained from the compatibility equation for the stress functions. The Galerkin procedure then provides a nonlinear differential equation for the time function, where the solution has been obtained by successive approximation method. Throughout the analysis it is assumed that, prior to the oscillatory motion, the shell is subjected to a deformation provided by large inplane uniformly distributed edge loads.

By rejecting the nonlinear term and specialising to the static case, the standard value of the buckling load is obtained and the result is verified for the isotropic case obtained by Nowacki [7] and Timoshenko [11]. Restitution of the nonlinear terms gives the post-buckling deformation of the shell.

Free nonlinear oscillations are studied in more detail, and the dependence of the frequency of oscillations on the magnitude of initial stress and the amplitude is discussed, for three types of anisotropy and various values of amplitude and initial stress.

Finally, the least critical loads have been calculated for different classes of anisotropy to exhibit the effects of large deflections and imperfections on elastic buckling.

\section{Equations and discussions}

Let the edges of the cylindrical shell be $x=0, x=a, y=0, y=b$. The origin of coordinates $(x, y)$ is located at a vertex of the middle plane of the shell. Let $x$ and $y$ be measured in the axial and the circumferential direction in the median surface of the undeformed cylindrical shell of thickness $h$ and made of rectilinearly orthotropic material whose axes of anisotropy are parallel to the edges of the shell. Let $u, v$ and $w$ be the components of displacement of a point on the median surface of the shell in the $x-, y-$ and radian directions respectively. Then at an arbitrary point in the median surface the unit strains in the $x$ - and $y$-directions, $\varepsilon_{x}, \varepsilon_{y}$ and the unit shear $\gamma_{x y}$, can be expressed in the following forms, including terms up to second 
order:

$$
\begin{aligned}
\varepsilon_{x} & =\frac{\partial u}{\partial x}+\frac{1}{2}\left(\frac{\partial w}{\partial x}\right)^{2}, \\
\varepsilon_{y} & =\frac{\partial v}{\partial y}+\frac{1}{2}\left(\frac{\partial w}{\partial y}\right)^{2}-\frac{w}{R}, \\
\gamma_{x y} & =\frac{\partial u}{\partial y}+\frac{\partial v}{\partial x}+\frac{\partial w}{\partial x} \cdot \frac{\partial w}{\partial y},
\end{aligned}
$$

where $R$ is the radius of the undeformed median surface of the shell. The orthotropic stresses and strains in the median surface of the shell are, however, related to each other by the following equations:

$$
\varepsilon_{x} E_{x}=\sigma_{x}-\nu_{1} \sigma_{y}, \quad \varepsilon_{y} E_{y}=\sigma_{y}-\nu_{2} \sigma_{x}, \quad \gamma_{x y}=\tau_{x y} / G .
$$

We assume that the shell is subjected to two sets of uniformly distributed inplane normal tractions $\sigma_{x}^{0}$ and $\sigma_{y}^{0}$ on the edges $x=0, a$ and $y=0, b$ respectively. These tractions produce a pure homogeneous deformation characterised by the unit extensions $\varepsilon_{x}^{0}$ and $\varepsilon_{y}^{0}$ which carry the shell into an initial state of plane stress. The edges of the shell are then placed on supports, the shell is loaded by a normal load $q(x, y, t)$ on its upper surface and set in flexural oscillatory motion. For definiteness, the edges of the shell are supposed to be freely supported, and either freely movable or prevented against displacements in the inplane directions.

It is further assumed that the transverse deflections of the shell are large; that is, of the order of the magnitude of the thickness $h$. While the geometrical nonlinearity creeps into the problem owing to the latter assumption, it is postulated that Hooke's law is valid in its orthotropic form.

By virtue of the formulae derived elsewhere by Donnell [3], the field equations, extended to a dynamic and rectilinearly orthotropic shell with an initial stress system, take the following form:

$$
\begin{aligned}
& \Lambda_{1}(w, \phi)=w_{x x x x}+2 l^{2} w_{x x y y}+k^{2} w_{y y y y}-v / D_{x}+\frac{\rho h}{D_{x}} w_{t t} \\
& -\frac{h}{D_{x}}\left[\left(\phi_{y y}+\sigma_{x}^{0}\right) w_{x x}+\phi_{x y} w_{x y}+\left(\phi_{x x}+\sigma_{y}^{0}\right) w_{y y}\right]-\frac{1}{R D_{x}} \phi_{x x}=0 \text {, } \\
& \Lambda_{2}(w, \phi)=\phi_{x x x x}+p^{2} \phi_{x x y y}+k^{2} \phi_{y y y y} \\
& -E_{y} \cdot h\left[\left(w_{x y}\right)^{2}-w_{x x} w_{y y}-\frac{1}{R} w_{x x}\right]=0,
\end{aligned}
$$

where $w$ is the deflection of the shell and $\phi$ is the stress function related to the membrane stresses generated by the large deflection of the shell (and marked by an asterisk) given by

$$
\sigma_{x}^{*}=\phi_{y y}, \quad \sigma_{y}^{*}=\phi_{x x}, \quad \sigma_{x y}^{*}=-\phi_{x y} .
$$


Furthermore

$$
k^{2}=\frac{D_{y}}{D_{x}}=\frac{E_{y}}{E_{x}}=\frac{\nu_{2}}{\nu_{1}}, \quad l^{2}=\nu_{1}+2 \frac{D_{x y}}{X_{x}}, \quad p^{2}=E_{y}\left(\frac{1}{G}-\frac{2 \nu_{1}}{E_{x}}\right),
$$

where $D_{x}$ and $D_{y}$ are bending rigidities in the $x$ and $y$ directions, $D_{x y}=$ $G h^{3} / 12$ and $E_{x}, E_{y}, G, \nu_{1}$ and $\nu_{2}$ Young's moduli, shear modulus and Poisson's ratios respectively. It should be noted here that the use of the stress function in (2.5) is equivalent to disregard of the inertia of inplane motion of the particles of median surface of the shell. However, this assumption is legitimate in this case, because the oscillations take place primarily in the transverse direction. It may be mentioned in this connection that (2.4) expresses the condition of compatibility between the stresses and strains. When $R \rightarrow \infty,(2.3)$ and (2.4) reduce to the corresponding equations for a flat plate derived elsewhere by Nowinski [8]. With a given distribution of the radial component of displacement $w,(2.4)$ gives the induced stresses in the median surface of the shell.

The solution for the deflection may be sought in the form of a trigonometric series of sine terms satisfying the boundary conditions imposed on the deflections,

$$
\begin{array}{lll}
w(x, y, t)=w_{x x}(x, t, t)=0 & \text { for } x=0, a & \text { and } 0 \leq y \leq b, \\
w(x, y, t)=w_{y y}(x, y, t)=0 & \text { for } y=0, b & \text { and } 0 \leq x \leq a,
\end{array}
$$

at any time.

Without loss of generality, let us confine ourselves to one term of the series only, and have an approximate solution of the form

$$
w(x, t, t)=\alpha_{m n} \sin \left(\beta_{m} x\right) \sin \left(\gamma_{n} y\right) \tau(t),
$$

where

$$
\beta_{m}=m \pi / a, \quad \gamma_{n}=n \pi / b,
$$

$\alpha_{m n}$ is a constant coefficient and $\tau(t)$ an unknown function of time to be determined later. This form satisfactorily describes the fundamental mode of oscillation which is of primary interest.

Introducing (2.8) into (2.4) we arrive at the following form of the stress function,

$$
\begin{aligned}
\phi= & \frac{E_{y}}{32} \alpha_{m n}^{2}\left[\frac{\beta_{m}^{2}}{k^{2} \gamma_{n}^{2}} \cos \left(2 \gamma_{n} y\right)+\frac{\gamma_{n}^{2}}{\beta_{n}^{2}} \cos \left(2 \beta_{m} x\right)\right] \tau^{2} \\
& +\frac{E_{y}}{R} \alpha_{m n} \frac{\beta_{m}^{1} \sin \left(\beta_{m} x\right) \sin \left(\gamma_{n} y\right)}{\beta_{m}^{4}+p^{2} \beta_{m}^{2} \gamma_{n}^{2}+k^{2} \gamma_{n}^{4}} \tau+c_{2}(t) \frac{x^{2}}{2}+c_{1}(t) \frac{y^{2}}{2} .
\end{aligned}
$$

The last two terms represent an integral of (2.4) obtained by posing $w=0$, and are adjoined to the solution in order to satisfy the boundary conditions 
for the membrane stresses (2.5). In view of (2.10), (2.5) yields

$$
\begin{aligned}
\sigma_{x}^{*}= & -\frac{1}{8} E_{x} \alpha_{m n}^{2} \beta_{m}^{2} \cos \left(2 \gamma_{n} y\right) \cdot \tau^{2} \\
& -\frac{E_{y}}{R} \frac{\alpha_{m n} \beta_{m}^{2} \gamma_{n}^{2} \sin \left(\beta_{m} x\right) \sin \left(\gamma_{n} y\right) \cdot \tau}{\left(\beta_{m}^{4}+p^{2} \beta_{m}^{2} \gamma_{n}^{2}+k^{2} \gamma_{n}^{4}\right)}+c_{1}(t), \\
\sigma_{y}^{*}= & -\frac{1}{8} E_{y} \alpha_{m n}^{2} \gamma_{n}^{2} \cos \left(2 \beta_{m} x\right) \cdot \tau^{2} \\
& -\frac{E_{y} \alpha_{m n} \beta_{m}^{4} \sin \left(\beta_{m} x\right) \sin \left(\gamma_{n} y\right) \cdot \tau}{\left(\beta_{m}^{4}+p^{2} \beta_{m}^{2} \gamma_{n}^{2}+k^{2} \gamma_{n}^{4}\right)}+c_{2}(t), \\
\sigma_{x y}^{*}= & \frac{E_{y} \alpha_{m n} \beta_{m}^{3} \gamma_{n} \cos \left(\beta_{m} x\right) \cos \left(\gamma_{n} y\right) \cdot \tau}{R\left(\beta_{m}^{4}+p^{2} \beta_{m}^{2} \gamma_{n}^{2}+k^{2} \gamma_{n}^{4}\right)} .
\end{aligned}
$$

With the help of (2.1) and (2.2), we can write

$$
\begin{aligned}
& \frac{\partial u}{\partial x}=\frac{1}{E_{x}}\left[\sigma_{x}^{*}-\nu_{1} \sigma_{y}^{*}\right]-\frac{1}{2}\left(\frac{\partial w}{\partial x}\right)^{2}, \\
& \frac{\partial v}{\partial y}=\frac{1}{E_{y}}\left[\sigma_{y}^{*}-\nu_{2} \sigma_{x}^{*}\right]-\frac{1}{2}\left(\frac{\partial w}{\partial y}\right)^{2}+\frac{w}{R} .
\end{aligned}
$$

Introducing (2.5), (2.8) and (2.10) into (2.12) and integrating the first equation of (2.12) with respect to $x$ from $x=0$ to $x=a$, and the second with respect to $y$ from $y=0$ to $y=b$, we can arrive at the following values of the relative displacements $\Delta x$ and $\Delta y$ for each pair of parallel edges of the shell produced by the membrane stresses (2.5).

$$
\begin{array}{r}
\Delta x=-\frac{1}{8} \alpha_{m n}^{2} \beta_{m}^{2} a \tau^{2}+\frac{a}{E_{x}}\left(c_{1}-\nu_{1} c_{2}\right) \\
-\frac{\alpha_{m n}}{R} \frac{\tau k^{2} \beta_{m}^{2}\left(\gamma_{n}^{2}-\nu_{1} \beta_{m}^{2}\right)}{\beta_{m}^{4}+p^{2} \beta_{m}^{2} \gamma_{n}^{2}+k^{2} \gamma_{n}^{4}}\left[\cos \left(\beta_{m} a\right)-1\right]\left[\cos \left(\gamma_{n} b\right)-1\right], \\
\Delta y=-\frac{1}{8} \alpha_{m n}^{2} \gamma_{n}^{2} b \tau^{2}+\frac{b}{E_{y}}\left(c_{2}-\nu_{2} c_{1}\right)+\frac{\alpha_{m n} \tau}{a \beta_{m} \gamma_{n}}\left[\cos \left(\beta_{m} a\right)-1\right] \\
\cdot\left[\cos \left(\gamma_{n} b\right)-1\right]\left[\frac{1}{R}-\frac{1}{R} \cdot \frac{\beta_{m}^{2}\left(\beta_{m}^{2}-\nu_{2} \gamma_{n}^{2}\right)}{\beta_{m}^{4}+p^{2} \beta_{m}^{2} \gamma_{n}^{2}+k^{2} \gamma_{n}^{4}}\right], \\
m, n=1,3,5, \ldots
\end{array}
$$

The mean stresses at the edges of the shell are

$$
c_{1}^{*}=c_{1}(t)-\frac{E_{y} h}{R} \frac{\alpha_{m n} \beta_{m}^{2} \gamma_{n}^{2}\left[\cos \left(\beta_{m} a\right)-1\right]\left[\cos \left(\gamma_{n} b\right)-1\right]}{\beta_{m}^{4}+p^{2} \beta_{m}^{2} \gamma_{n}^{2}+k^{2} \gamma_{n}^{4}} \tau
$$

and

$$
c_{2}^{*}=c_{2}(t)-\frac{E_{y} h}{R} \frac{\alpha_{m n} \beta_{m}^{4}\left[\cos \left(\beta_{m} a\right)-1\right]\left[\cos \left(\gamma_{n} b\right)-1\right]}{\beta_{m}^{4}+p^{2} \beta_{m}^{2} \gamma_{n}^{2}+k^{2} \gamma_{n}^{4}} \tau .
$$


Let us assume that the resistance of the supports against an inplane normal displacement is in direct proportion to the mean membrane stresses by which they are acted upon. In other words, let us pose

$$
\Delta x=c_{1}^{*}(t) h / \beta_{1}^{*}, \quad \Delta y=c_{2}^{*}(t) h / \beta_{2}^{*},
$$

where $\beta_{1}^{*}$ and $\beta_{2}^{*}$ are spring constants of the supports along the edges $x=0$, $a$ and $y=0, b$, respectively. Apparently, we have

$$
\beta_{i}^{*}=\infty, \quad i=1,2,
$$

for a support preventing any normal displacement, and

$$
c_{1}^{*}=0=c_{2}^{*} \text {. }
$$

for freely movable edges.

From (2.13), (2.14) and (2.15), we find the value of the parametric functions $C_{i}(t), i=1,2$, in the form

$$
c_{i}=\beta_{i} \alpha_{m n}^{2} \tau^{2}-\beta_{i} \alpha_{m n} \tau,
$$

where

and

$$
\begin{aligned}
\beta_{1} & =\frac{E_{x}}{8 \delta}\left[\nu_{2} \gamma_{n}^{2}+\beta_{m}^{2}\left(1-E_{y} h / b \beta_{2}^{*}\right)\right], \\
\beta_{2} & =\frac{E_{y}}{8 \delta}\left[\nu_{1} \beta_{m}^{2}+\gamma_{n}^{2}\left(1-E_{x} h / a \beta_{1}^{*}\right)\right], \\
\delta & =\left[1-E_{x} h / a \beta_{1}^{*}\right]\left[1-E_{y} h / b \beta_{2}^{*}\right]-\nu_{1} \nu_{2}, \\
\beta_{1}^{\prime} & =\frac{E_{x}}{\delta}\left[\frac{1}{a}\left(1-E_{y} h / b \beta_{2}^{*}\right) C_{m n}+\frac{\nu_{2}}{b} D_{m n}\right], \\
\beta_{2}^{\prime} & =\frac{E_{y}}{\delta}\left[\frac{1}{b}\left(1-E_{x} h / a \beta_{1}^{*}\right) D_{m n}+\frac{\nu_{1}}{a} C_{m n}\right],
\end{aligned}
$$

$$
\begin{gathered}
C_{m n}=\frac{\left[\cos \left(\beta_{m} a\right)-1\right]\left[\cos \left(\gamma_{n} b\right)-1\right] \beta_{m}^{2}}{b \beta_{m} \gamma_{n}\left(\beta_{m}^{4}+p^{2} \beta_{m}^{2} \gamma_{n}^{2}+k^{2} \gamma_{n}^{4}\right) R}\left[\frac{E_{y} h \gamma_{n}^{2}}{\beta_{1}^{*}}-k^{2}\left(\gamma_{n}^{2}-\nu_{1} \beta_{m}^{2}\right)\right], \\
D_{m n}=\left[\cos \left(\beta_{m} a\right)-1\right]\left[\cos \left(\gamma_{n} b\right)-1\right]\left[\frac{E_{y} h \beta_{m}^{4} / a b \beta_{m} \gamma_{n}}{R \beta_{2}^{*}\left(\beta_{m}^{4}+p^{2} \beta_{m}^{2} \gamma_{n}^{2}+k^{2} \gamma_{n}^{4}\right)}\right. \\
\left.-\frac{1}{a R \beta_{m} \gamma_{n}} \cdot\left\{1+\frac{\beta_{m}^{2}\left(\beta_{m}^{2}-\nu_{2} \gamma_{n}^{2}\right)}{\beta_{m}^{4}+p^{2} \beta_{m}^{2} \gamma_{n}^{2}+k^{2} \gamma_{n}^{4}}\right\}\right], \\
m, n=1,3,5, \ldots
\end{gathered}
$$

Let us note that the nonlinearity of the problem is associated with the magnitude of the deflection and not with the pinning of the edges. This fact is in contrast with what we find in oscillations of beams.

Let us now return to the remaining field equation $(2.3), \Lambda_{1}(w, \phi)=0$ and proceed in the following fashion for its evaluation. For the investigation of 
free vibrations of the shell solely, we disregard the load term $q(x, y, t)$ in (2.3). Then introducing (2.8) and (2.10) into (2.3), we apply the procedures of Galerkin; that is, (2.3) is multiplied by the spatial part of the deflection (2.8), and the result is integrated over the domain of the shell.

A lengthy but elementary calculation leads either to a trivial solution $\alpha_{m n}=0$ (which has to be rejected), or to the following well known noniinear differentiai equation of Duffing type for the unknown time function $\tau$,

$$
\ddot{\tau}+\Gamma_{1}^{*} \tau-\Gamma_{2}^{*} \tau^{2}+\Gamma_{3}^{*} \tau^{3}=0
$$

where

$$
\begin{gathered}
\Gamma_{1}^{*}=\frac{D_{x}}{\rho h}\left\{\left(\beta_{m}^{4}+2 l^{2} \beta_{m}^{2} \gamma_{n}^{2}+k^{2} \gamma_{n}^{4}\right)+\frac{h}{D_{x}}\left(\sigma_{x}^{0} \beta_{m}^{2}+\sigma_{y}^{0} \gamma_{n}^{2}\right)\right. \\
\left.+\frac{E_{y} h}{R^{2} D_{x}}\left[\frac{\beta_{m}^{4}}{\beta_{m}^{4}+p^{2} \beta_{m}^{2} \gamma_{n}^{2}+k^{2} \gamma_{n}^{4}}\right]\right\}, \\
\Gamma_{2}^{*}=\frac{8 \cdot D_{x} \gamma_{m n}}{a b \rho h}\left[\frac { h } { D _ { x } } \left\{\beta_{m}^{4} \gamma_{n}^{2} E_{y} \frac{\left[\cos ^{3}\left(\beta_{m} a\right)-1\right]\left[\cos ^{3}\left(\gamma_{n} b\right)-1\right]}{9 \beta_{m} \gamma_{n} R\left(\beta_{m}^{4}+p^{2} \beta_{m}^{2} \gamma_{n}^{2}+k^{2} \gamma_{n}^{4}\right)}\right.\right. \\
+\beta_{m}^{4} \gamma_{n}^{2} E_{y}\left[\frac{\cos \left(3 \beta_{m} a\right)-1}{3 \beta_{m}}-3 \cdot \frac{\left\{\cos \left(\beta_{m} a\right)-1\right\}}{\beta_{m}}\right] \\
\left.\cdot \frac{\left[\frac{\cos \left(3 \gamma_{n} b\right)-1}{3 \gamma_{n}}-\frac{3\left(\cos \left(\gamma_{n} b\right)-1\right\}}{\gamma_{n}}\right]}{16 R\left(\beta_{m}^{4}+p^{2} \beta_{m}^{2} \gamma_{n}^{2}+k^{2} \gamma_{n}^{4}\right)}\right\} \\
\left.+\frac{4 E_{y} \gamma_{n}^{2} a b}{256 R D_{x}}+h a b\left(\beta_{1}^{\prime} \beta_{m}^{2}+\beta_{2}^{\prime} \gamma_{n}^{2}\right) / 8 D_{x}\right],
\end{gathered}
$$

It may be noted in this connection that as $R \rightarrow \infty$ the expressions for $\Gamma_{1}^{*}$ and $\Gamma_{3}^{*}$ reduces to those of the forms $\Gamma_{1}$ and $\Gamma_{3}$ obtained elsewhere by Nowinski [8] in the case of an anisotropic plate and $\Gamma_{2}^{*} \rightarrow 0$. The solution of (2.22) can be obtained in the form of an incomplete elliptic integral [5, $10]$, but such a solution is complicated and not suitable for any practical application and, therefore, a method of successive approximation [5] has been used to obtain the required solution.

Let us substitute

$$
\xi=\sqrt{\Gamma_{1}^{*}} \cdot t
$$

in (2.22), which reduces to

$$
\frac{d^{2} \tau}{d \xi^{2}}+\tau+f_{2} \tau^{2}+f_{3} \tau^{3}=0
$$


where

$$
f_{2}=-\Gamma_{2}^{*} / \Gamma_{1}^{*} \quad \text { and } \quad f_{3}=\Gamma_{3}^{*} / \Gamma_{1}^{*} \text {. }
$$

Now, using the transformation

$$
\zeta=\sqrt{1+\beta} \cdot \xi
$$

(2.25) reduces to

$$
(1+\beta) \frac{d^{2} \tau}{d \xi^{2}}+\tau=-f_{2} \tau^{2}-f_{3} \tau^{3}
$$

Let $\tau_{1}$ and $-\tau_{2}$ be the maximum and minimum amplitudes respectively of $\tau$ then, $\beta$ and $\tau$ are expanded in power series of $\tau_{2}$ in the following forms:

$$
\begin{gathered}
\beta=-\beta_{1} \tau_{2}+\beta_{2} \tau_{2}^{2}-\beta_{3} \tau_{2}^{3}+\cdots \\
\tau=-\eta_{1}(\zeta) \tau_{2}+\eta_{2}(\xi) \tau_{2}^{2}-\eta_{3}(\xi) \tau_{2}^{3}+\cdots
\end{gathered}
$$

Substituting (2.29) and (2.30) in (2.28) and equating coefficients of like powers of $\tau_{2}$ on both sides of (2.28), we obtain a set of differential equations in $\eta_{1}, \eta_{2}, \eta_{3}, \ldots$ associated with $\beta_{1}, \beta_{2}, \beta_{3}, \ldots$ Solving these equations by the successive approximation method $[9,10]$ under the initial conditions $\eta_{1}(0)=1, \eta_{2}(0)=\eta_{3}(0)=\cdots=0$ and $\dot{\eta}_{1}(0)=\dot{\eta}_{2}(0)=\dot{\eta}_{3}(0)=\cdots=0$, we can obtain the values of $\eta_{1}, \eta_{2}, \eta_{3}, \ldots$ and $\beta_{1}, \beta_{2}, \beta_{3}, \ldots$ Thus the solution of $(2.28)$ becomes

$$
\begin{aligned}
\tau= & {\left[-\frac{1}{2} f_{2} \tau_{2}^{2}+\frac{1}{3} f_{2}^{2} \tau_{2}^{3}-\left(\frac{25}{48} f_{2}^{3}-\frac{21}{32} f_{2} f_{3}\right) \tau_{2}^{4}\right.} \\
& \left.+\left(\frac{25}{36} f_{2}^{4}-\frac{29}{34} f_{2}^{2} f_{3}\right) \tau_{2}^{5}-\ldots\right] \\
& +\left[-\tau_{2}+\frac{1}{3} f_{2} \tau_{2}^{2}-\left(\frac{29}{144} f_{2}^{2}-\frac{1}{32} f_{3}\right) \tau_{2}^{3}+\left(\frac{119}{432} f_{2}^{3}-\frac{35}{96} f_{2} f_{3}\right) \tau_{2}^{4}\right. \\
& \left.+\left(\frac{6971}{2-736} f_{2}^{4}-\frac{1475}{2304} f_{2}^{2} f_{2}+\frac{23}{1024} f_{2}^{2}\right) \tau_{2}^{5}+\ldots\right] \cos \zeta \\
& +\left[-\left(\frac{1}{48} f_{2} \tau_{2}^{2}-\frac{1}{9} f_{2}^{2} \tau_{2}^{3}+\left(\frac{2}{9} f_{2}^{3}-\frac{1}{32} f_{2} f_{3}\right) \tau_{2}^{4}\right) \tau_{3}^{3}+\left(\frac{1}{48} f_{2}^{3}+\frac{1}{32} f_{2} f_{3}\right) \tau_{2}^{4}\right. \\
& \left.-\left(\frac{31}{576} f_{2}^{4}-\frac{11}{384} f_{2}^{2} f_{3}-\frac{1}{128} f_{3}^{2}\right) \tau_{2}^{5} \cdots\right] \cos 3 \zeta \\
& +\ldots \quad(2.31)
\end{aligned}
$$

Then (2.31) is the solution of (2.28) when the amplitude of vibration is expressed as the function of $\zeta$ and $\tau_{2}$. For the case of infinitesimal value 
of $\tau_{2}$, the constant term and the higher harmonic terms in (2.31) can be neglected except only the fundamental harmonic term which is the solution of the linear theory. Then $(2.31)$ is the periodic function with respect to $\zeta$ with the period $2 \pi$.

The period of the motion is given by

$$
\begin{aligned}
T^{*}(t)=\frac{2 \pi}{\sqrt{\Gamma_{1}^{*}}}[1+ & \left(\frac{5}{12} f_{2}^{2}-\frac{3}{8} f_{2}\right) \tau_{2}^{2}-\left(\frac{5}{18} f_{2}^{3}-\frac{1}{4} f_{2} f_{3}\right) \tau_{2}^{3} \\
& \left.+\left(\frac{385}{576} f_{2}^{4}-\frac{275}{192} f_{2}^{2} f_{3}+\frac{57}{256} f_{3}^{2}\right) \tau_{2}^{4}-\ldots\right]
\end{aligned}
$$

Equations (2.31) and (2.32) give the amplitude and period of nonlinear vibration of the above mentioned shell. The period is a function of amplitude, which is the characteristic of the nonlinear vibration, and the relation between the maximum and minimum values of the amplitudes $\tau_{1}$ and $-\tau_{2}$, is given below.

Applying the so-called energy integral to (2.25), the following equation is obtained:

$$
\left(\frac{d \tau}{d \xi}\right)^{2}+\tau^{2}+\frac{2}{3} f_{2} \tau^{2}+\frac{1}{2} f_{3} \tau^{4}=2 E_{T}
$$

where $E_{T}$ is the total energy of the vibrating system. For extremum, $\frac{d \tau}{d \xi}=0$ at $\tau=\tau_{1},-\tau_{2}$, and this condition reduces (2.33) to

$$
\tau_{1}^{2}\left(1+\frac{2}{3} f_{2} \tau_{1}+\frac{1}{2} f_{3} \tau_{1}^{2}\right)=\tau_{2}^{2}\left(1-\frac{2}{3} f_{2} \tau_{2}+\frac{1}{2} f_{2} \tau_{2}^{2}\right)=2 E_{T}
$$

where $\tau_{1}$ and $\tau_{2}$ can be determined independently whenever $E_{T}$ is given in accordance with the initial conditions.

\section{Free liner oscillations}

The circular frequency of the linear oscillations of the orthotropic shell is obtained from (2.32) by putting $\tau_{2}=0$, which yields

$$
\omega_{0}=\sqrt{\Gamma_{i}^{*}}
$$


If we are concerned with the fundamental mode $(m=n=1)$ and leave out the initial stresses, then

$$
\begin{aligned}
\omega_{0}=\pi^{2}\left(\frac{D_{x}}{\rho h}\right)^{1 / 2}\left[\frac{1}{b^{4}}\left\{\left(\frac{b}{a}\right)^{4}+2 l^{2}\left(\frac{b}{a}\right)^{2}+k^{2}\right\}\right. \\
\left.+\frac{h E_{y}(b / a)^{4}}{R^{2} D_{x}\left\{\left(\frac{b}{a}\right)^{4}+p^{2}\left(\frac{b}{a}\right)^{2}+k^{2}\right\}}\right]^{1 / 2} .
\end{aligned}
$$

As $R \rightarrow \infty,(3.2)$ reduces to that of an anisotropic plate as obtained by Nowinski [8].

\section{Buckling load}

Let us pose $\alpha_{m n}=0$ and $\tau=1$ in (2.22), and ask for the critical compressive load

$$
P_{x}=-h \sigma_{x}^{0}, \quad P_{y}=-h \sigma_{y}^{0} .
$$

Evidently (2.22) now yields the condition $\Gamma_{1}^{*}=0$, or, more explicitly,

$$
\begin{aligned}
\beta_{m}^{2}\left(P_{x}\right)_{\text {crit }}+\gamma_{n}^{2}\left(P_{y}\right)_{\text {crit }}= & D_{x}\left(\beta_{m}^{4}+2 l^{2} \beta_{m}^{2} \gamma_{n}^{2}+k^{2} \gamma_{n}^{4}\right) \\
& +\frac{E_{y} h}{R^{2}}\left(\frac{\beta_{m}^{4}}{\beta_{m}^{4}+p^{2} \beta_{m}^{2} \gamma_{n}^{2}+k^{2} \gamma_{n}^{4}}\right) .
\end{aligned}
$$

If we put $k^{2}=l^{2}=1, a=b, p^{2}=2, P_{y}=0,(4.2)$ reduces to

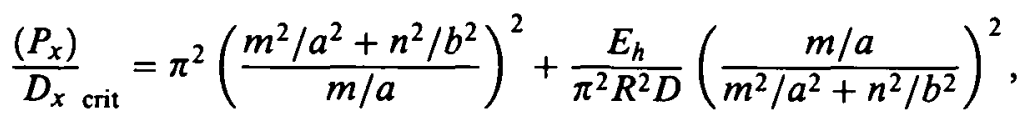

which was derived elsewhere in $[7,11]$ and the least critical load can be obtained from (4.3) as

$$
\left[\left(P_{x} / h\right) \mathrm{crit}\right] \min =\frac{E_{h}}{R \sqrt{3\left(1-\nu^{2}\right)}}
$$


considering the left hand side to be a continuous function of $\left[\left(\mathrm{m}^{2} / \mathrm{a}^{2}+\right.\right.$ $\left.\left.n^{2} / b^{2}\right) /(m / a)\right]^{2}$.

\section{Post buckling stage}

The post buckiing criticai behaviour of the orihoiropic shieli cân easilì be described by letting $\tau=1$ in (2.22). We have then

$$
\left(\frac{A}{h}\right)^{2} \Gamma_{3}^{*}-\left(\frac{A}{h}\right) \Gamma_{2}+\Gamma_{1}^{*}=0, \quad \text { where } \alpha_{m n}=A
$$

and $\Gamma_{2}=\Gamma_{2}^{*} /\left(\alpha_{m n} / h\right)$.

When $R \rightarrow \infty, \Gamma_{n} \rightarrow 0$ and the result is the same as that obtained by Nowinski [8] in the case of anisotropic plates. The relation (5.1) will help in the determination of maximum deflection of the shell.

To proceed further let us pose $k^{2}=l^{2}=1$ and assume for definiteness that the edges $x=0, a$ of the shell are freely movable in the normal directions, while the edges $y=0, b$ are rigidly pinned. Again, the active loading of the shell consists of the uniformly distributed forces $P_{x}$ only. This yields $\beta_{1}^{*}=0$, $\beta_{2}^{*}=\infty, c_{1}^{*}=0$. Hence we have $\beta_{1} \rightarrow 0$ and $\beta_{2} \rightarrow \pi^{2} E / 8 b^{2}$,

$$
\begin{aligned}
& \beta_{1}^{\prime}=-\frac{E}{R}\left(\frac{b}{a}\right)^{2} \frac{4}{\left(1+b^{2} / a^{2}\right)^{2}}, \\
& \beta_{2}^{\prime}=-\frac{4 E}{R}\left[\frac{1}{\pi^{2}}\left\{1-\left(\frac{b}{a}\right)^{2} \frac{(b / a)^{2}-\nu_{2}}{\left[1+(b / a)^{2}\right]^{2}}\right\}+\nu_{1}\left(\frac{b}{a}\right)^{2} \frac{1}{\left(1+b^{2} / a^{2}\right)^{2}}\right],
\end{aligned}
$$

and we have then, from (5.1),

$$
\frac{A}{h}=\frac{1 \Gamma_{2}}{2 \Gamma_{3}^{*}} \pm\left[\left(\frac{\Gamma_{2}}{\Gamma_{3}^{*}}\right)^{2}-4\left(\frac{\Gamma_{1}^{*}}{\Gamma_{3}^{*}}\right)\right]^{1 / 2}
$$

For the deflection to be real, we have $\left(\Gamma_{2} / \Gamma_{3}^{*}\right)^{2} \geq 4\left(\Gamma_{1}^{*} / \Gamma_{3}^{*}\right)$. The maximum deflection can be easily obtained from (5.2).

\section{Free nonlinear oscillations}

Let us now turn to the investigation of free nonlinear oscillations of the shell, to which we attribute different degrees of anisotropy. Before doing this, let us find out the equation for the critical load of the shell by assuming that, throughout the entire period of deformations, the normal forces acting on the edges of the shell are related to each other, so that

$$
\sigma_{y}^{0}=\alpha \sigma_{x}^{0}
$$


where $\alpha$ is a preassigned constant parameter. From (4.2) we can write

$$
\begin{aligned}
\left(\sigma_{x}^{0}\right)_{\text {crit }}= & \frac{D_{x} \beta_{m}^{4}+2 l^{2} \beta_{m}^{2} \gamma_{n}^{2}+k^{2} \gamma_{n}^{4}}{h\left(\beta_{m}^{2}+\alpha \gamma_{n}^{2}\right)} \\
& +\frac{E_{y} \beta_{m}^{4}}{R^{2}\left(\beta_{m}^{2}+\alpha \gamma_{n}^{2}\right)\left(\beta_{m}^{4}+\beta^{2} \beta_{m}^{2} \gamma_{n}^{2}+k^{2} \gamma_{n}^{4}\right)},
\end{aligned}
$$

and therefore for $m=n=1$,

$$
\begin{aligned}
& \Gamma_{1}^{*}= {\left[1+\frac{\sigma_{x}^{0}}{\left(\sigma_{x}^{0}\right)_{\text {crit }}}\right] \frac{D_{x}}{f_{h}}\left(\frac{\pi}{a}\right)^{4}, } \\
& \frac{\left[\left\{1+2 l^{2}\left(\frac{a}{b}\right)^{2}+k^{2}\left(\frac{a}{b}\right)^{4}\right\}^{2}+\left(\frac{1}{\pi}\right)^{4}\left(\frac{k}{h R}\right)^{2} 12\left(1-\nu_{1} \nu_{2}\right)\right]}{1+p^{2}\left(\frac{a}{b}\right)^{2}+k^{2}\left(\frac{a}{b}\right)^{4}}, \\
& \Gamma_{2}^{*}=\frac{24}{a b} \frac{D_{x} a k^{2}\left(1-\nu_{1} \nu_{2}\right)}{\rho h b h^{2} R} \cdot \frac{\left[\frac{80}{9}+\frac{\pi^{2}}{16}\left\{1+p^{2}\left(\frac{a}{b}\right)^{2}+k^{2}\left(\frac{a}{b}\right)^{4}\right\}+2\left\{p^{2}\left(\frac{a}{b}\right)^{2}+k^{2}\left(\frac{a}{b}\right)^{4}-1\right\}\right]}{1+p^{2}\left(\frac{a}{b}\right)^{2}+k^{2}\left(\frac{a}{b}\right)^{4}}, \\
& \Gamma_{3}^{*}=\frac{D_{x}}{\rho}\left(\frac{\pi}{a}\right)^{4}\left[1+\frac{9}{8} k^{2}\left(\frac{a}{b}\right)^{4}\right] .
\end{aligned}
$$

Consequently $f_{2}$ and $f_{3}$ can be written as $(m=n=1)$

$$
\begin{aligned}
& f_{2}= \frac{24\left(1-\nu_{1} \nu_{2}\right) k^{2}\left(\frac{a}{b}\right)^{2}}{\frac{h R}{a a} \pi^{4}\left[1+\sigma_{x}^{0} /\left(\sigma_{x}^{0}\right)_{\text {crit }}\right]} \\
& \cdot\left[\frac{\frac{80}{9}+\pi^{2}\left\{1+p^{2}\left(\frac{a}{b}\right)^{2}+k^{2}\left(\frac{a}{b}\right)^{4}\right\}+2\left\{p^{2}\left(\frac{a}{b}\right)^{2}+k^{2}\left(\frac{a}{b}\right)^{4}-1\right\}}{\frac{12\left(1-\nu_{1} \nu_{2}\right) k^{2}}{\pi^{4}\left(\frac{b}{a}\right)^{2}\left(\frac{B}{a}\right)^{2}}+\left\{1+p^{2}\left(\frac{a}{b}\right)^{2}+k^{2}\left(\frac{a}{b}\right)^{4}\right\} \cdot\left\{1+2 l^{2}\left(\frac{a}{b}\right)^{2}+k^{2}\left(\frac{a}{b}\right)^{4}\right\}}\right]^{(6.6)} \\
& f_{3}=\frac{12\left(1-\nu_{1} \nu_{2}\right)\left\{1+\frac{9}{8} k^{2}\left(\frac{a}{b}\right)^{4}\right\} \cdot\left\{1+p^{2}\left(\frac{a}{b}\right)^{2}+k^{2}\left(\frac{a}{b}\right)^{4}\right\}}{\left.\left[1+\sigma_{x}^{0} /\left(\sigma_{x}^{0}\right)_{\text {crit }}\right]\left\{1+p^{2}\left(\frac{a}{b}\right)^{2}+k^{2}\left(\frac{a}{b}\right)^{4}\right\}\left\{a+2 l^{2}\left(\frac{a}{b}\right)^{2} k^{2}\left(\frac{a}{b}\right)^{4}\right\}+\frac{k^{2}}{\pi^{4}}\left(\frac{a}{b}\right)^{2} \cdot\left(\frac{a}{R}\right)^{2} \cdot 12\left(1-\nu_{1} \nu_{2}\right)\right]} .
\end{aligned}
$$

The frequency of nonlinear oscillations appears now as

$$
\omega^{*}=\sqrt{\Gamma_{1}^{*}}\left[1-\left\{\frac{5}{12}\left(\frac{\Gamma_{2}^{*}}{\Gamma_{1}^{*}}\right)^{2}-\frac{3}{8}\left(\frac{\Gamma_{3}^{*}}{\Gamma_{1}^{*}}\right)\right\}\left(\tau_{2}^{\prime}\right)^{2}+\ldots\right],
$$

where $\tau_{2}^{\prime}=\alpha_{11} \tau_{2} / h$.

For initial compressive stress, i.e., $\sigma_{x}^{0}<0$, the frequency may vanish. This happens when

$$
\left[1-\frac{\sigma_{x}^{0}}{\left(\sigma_{x}^{0}\right)_{\text {crit }}}\right]^{2}-\left[\frac{5}{12} f_{2}^{2}-\frac{3}{8} f_{3}\left(1-\frac{\sigma_{x}^{0}}{\left(\sigma_{x}^{0}\right)_{\text {crit }}}\right) \tau_{2}^{2}\left(\frac{\alpha_{11}}{h}\right)\right]^{2} \simeq 0,
$$

for the first approximation in the expansion. 


\section{Numerical results and discussions}

Apparently, the larger the amplitude of oscillations the larger inplane stress which brings the motion to a pause. The relation is displayed on graphs in Figure 1 for different types of anisotropy as illustrated in Table 1.

It is noted that in the entire range of variability of the ratio $\sigma_{x}^{0} /\left(\sigma_{x}^{0}\right)_{\text {crit }}$, starting from the horizontai ine $\sigma_{x}^{0} /\left(\sigma_{x}^{0}\right)_{\text {crit }}=i$ up to the correlaied currve, the nonlinear periodic motion subsists despite the fact that the load acting on

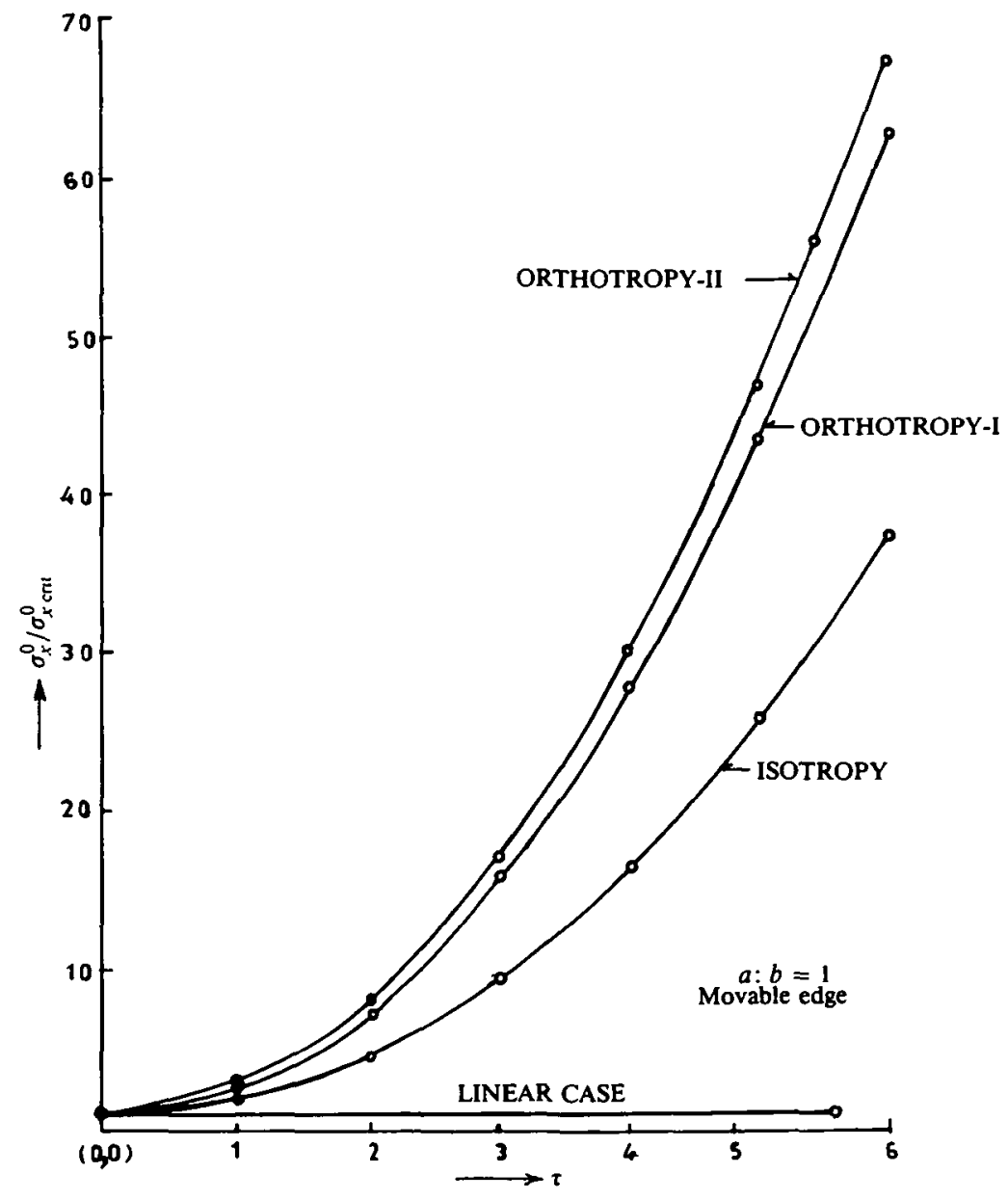

FIGURE 1. Initial compression versus amplitude for a vanishing frequency of nonliner oscillations. 
TABLE 1

\begin{tabular}{|l|c|c|c|c|c|}
\hline Type & $\nu_{1}$ & $\nu_{2}$ & $k^{2}$ & $l^{2}$ & $p^{2}$ \\
\hline Orthotropy I & .05 & .025 & .50 & .2228 & 1.895 \\
\hline Orthotropy II & .20 & .010 & .05 & .1078 & 1.665 \\
\hline Isotropy III & .30 & .300 & 1.00 & 1.0000 & 1.000 \\
\hline
\end{tabular}

the shell exceeds the critical value. While both types of orthotropy listed in the table differ considerably from each other their influence on the cessation of the oscillations differs only slightly.

From (3.2) and (6.8), $\omega^{*} / \omega_{0}>0$ for the first approximation if $\sigma_{x}^{0}(>0)$ is a tensile stress and $\frac{3}{8} f_{3} \gg \frac{5}{12} f_{2}$.

To illustrate the influence of the initial stress on the frequency of nonlinear oscillations Figure 2 (i, ii) depicts the dependence of the relative frequency $\omega^{*} / w_{0}^{*}\left(w_{0}^{*}\right.$ is the frequency of nonlinear oscillations of a shell free from initial stress) on the ration $\sigma_{x}^{0} /\left(\sigma_{x}^{0}\right)_{\text {crit }}$. With an increasing tensile stress we observe the well-known fact of increase of the frequency.

Figure 3 (i-iv) displays the relation between the relative frequency and the relative amplitude for various values of compressive stresses $k=\sigma_{x}^{0} /\left(\sigma_{x}^{0}\right)_{\text {crit }}$. An inspection of the curves reveals that with an increasing initial compression, and the values of the amplitude kept unchanged, the frequency of the oscillations decreases.

\section{B. Minimum buckling loads due to various classes of anisotropy}

Equation (4.2) has been simplified in the following form to obtain the buckling loads due to various classes of anisotropy:

$$
\begin{aligned}
\frac{\left(P_{x}\right)}{D_{x} \text { crit }}+\frac{\left(P_{y}\right)}{D_{x} \text { crit }} \cdot\left(\frac{n a}{m b}\right)^{2}= & \left(\frac{n \pi}{b}\right)^{2} \cdot\left[\lambda^{2}+2 l^{2}+\frac{k^{2}}{\lambda^{2}}\right] \\
& +\frac{E_{y} \cdot h}{D_{x} \cdot R^{2}}\left(\frac{b}{n \pi}\right)^{2} \cdot \frac{1}{\left[\lambda^{2}+p^{2}+\frac{k^{2}}{\lambda^{2}}\right]},
\end{aligned}
$$

where $\lambda=\beta_{m} / \gamma_{n}=m b /(n a)$. If $\sigma_{y}^{0}=0$ and $n=1$, then (7.1) reduces to

$$
\psi={\frac{\left(P_{x}\right)}{D_{x}}}^{\text {crit }}=\left(\frac{\pi}{b}\right)^{2}\left[z+2 l^{2}\right]+\frac{E_{y} \cdot h}{D_{x} \cdot R^{2}}\left(\frac{b}{\pi}\right)^{2} \frac{1}{\left[z+p^{2}\right]},
$$

where $z=\lambda^{2}+k^{2} / \lambda^{2}$. The least value of $\psi$ will be obtained as

$$
\psi_{\min }=\frac{4}{h R} \sqrt{\frac{E_{y}}{E_{x}}} \sqrt{3\left(1-\nu_{1} \nu_{2}\right)}+\left(\frac{\pi}{b}\right)^{2}\left[2 l^{2}-p^{2}\right],
$$

considering $\psi$ to be a continuous function of $z$. 

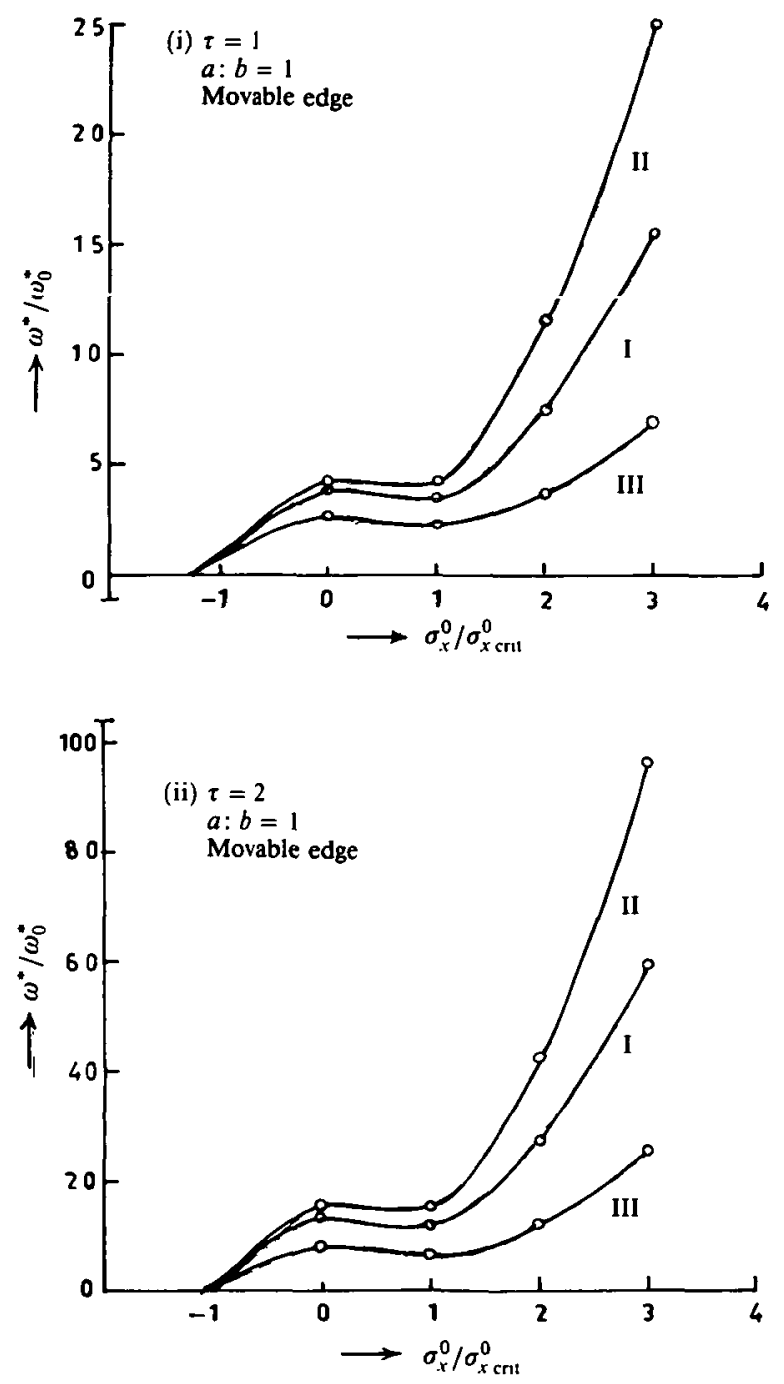

FIGURE 2. Nonlinear frequency versus initial stress for various amplitude and classes of anisotropy.

If $E_{x}=E_{y}, \nu_{1}=\nu_{2}, p^{2}=2$ and $l^{2}=1$, we get back the result (4.4) from (7.3), and this is true for all values of $n$. Moreover, we write.

$$
\chi_{\min }=h^{2}\left(\psi_{\min }\right)=\frac{4 h}{R} \sqrt{\frac{\nu_{2}}{\nu_{1}}} \cdot \sqrt{3\left(1-\nu_{1} \nu_{2}\right)}-\pi^{2}\left(\frac{h}{b}\right)^{2}\left(2 l^{2}-p^{2}\right)
$$

Hence, for different cases of anisotropy, the values of $\chi_{\min }$ can be obtained from (7.4) and are presented in Table 2. 
TABLE 2

\begin{tabular}{|l|c|c|c|c|c|c|c|c|}
\hline Type & $\nu_{1}$ & $\nu_{2}$ & $k^{2}$ & $l^{2}$ & $p^{2}$ & $h / b$ & $h / R$ & $\chi_{\min }$ \\
\hline Orthotropy I & 0.05 & 0.025 & 0.50 & 0.2228 & 1.895 & 0.1 & 0.1 & 0.3454 \\
\hline Orthotropy II & 0.20 & 0.010 & 0.05 & 0.1078 & 1.6665 & 0.1 & 0.1 & 0.0116 \\
\hline Isotropy & 0.30 & 0.300 & 1.00 & 1.000 & 1.000 & 0.1 & 0.1 & 0.65 \\
\hline
\end{tabular}

If $\sigma_{x}^{0}=0$, similar results can be obtained for $\left(P_{y}\right)_{\text {crit }}$ from (7.1).
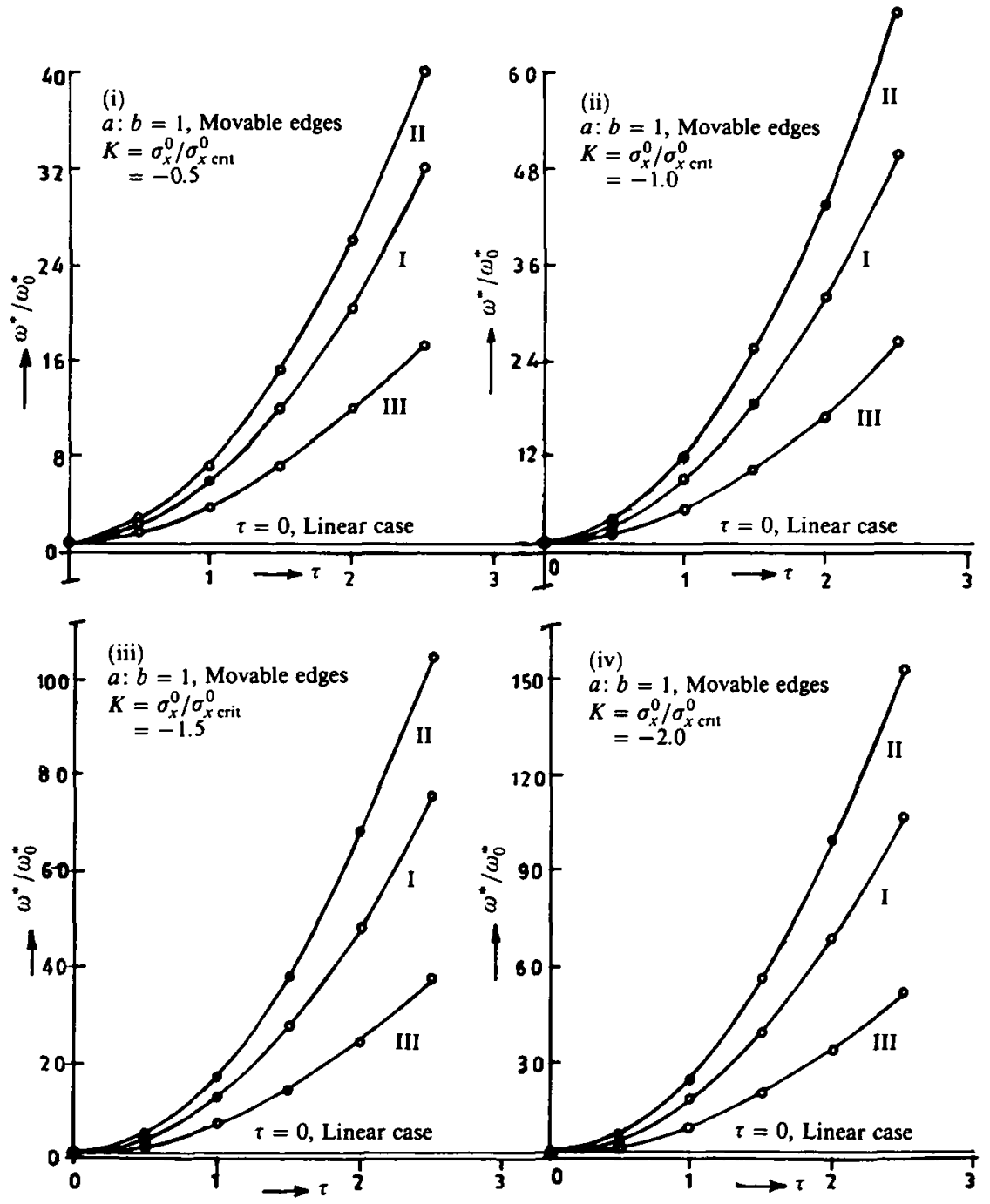

FIGURE 3. Frequency versus amplitude for various ratios of initial compression. 


\section{Acknowledgement}

I take this opportunity to express my thanks to the referee for his valuable comments for the improvement of the paper.

\section{References}

[1] A. Ambartsumian and A. A. Khachaturian, "On the stability of vibrations of anisotropic plates”, Izv. Akad. Nauk SSSR, Old. Tekhn., Nauk (1960) 113-122.

[2] V. V. Bolotin, The dynamic stability of elastic systems (English translation), (Holden Day, San Francisco, 1964).

[3] L. H. Donnell, "A new theory for the buckling of thin cylinders under axial compression and bending", Trans. Amer. Soc. Mech. Eng. 56 (1934) 795-815.

[4] R. M. Evan-Iwanowski, "On the parametric response of structures", Appl. Mech. Rev., 18 (1965) 699-702.

[5] G. Z. Harris and E. H. Mansfield, "On the large deflection vibrations of elastic plates", Philos. Trans. Roy. Soc. London Ser. A 261 (1967) 289-300.

[6] S. G. Lekhnitsky, Anisotropic plates (English translation), (Gordon and Breach Science Publishers, New York, 1956).

[7] W. Nowacki, "Problems of the stability and free vibrations of a cylindrical shell", App. Mech. 3 (1955) 17-23.

[8] J. L. Nowinski, "Nonlinear oscillations and stability of plates under large initial stress", Tech. Rep. 51 (Mechn. Eng. Dept., University of Delaware, Newark, Delaware, 1965), 1-20.

[9] M. Sunakawa, "Influences of temperature change and large amplitude on free flexural vibration of rectangular elastic plates", Tokyo University ISAS Rep. 402, 31 (1966) 3-15.

[10] S. P. Timoshenko, Vibration problems in engineering (D. Van Nostrand Company Inc., 1961) 270-278.

[11] S. P. Timoshenko and J. M. Gere, Theory of elastic stability (McGraw-Hill Book Company Inc., Second ed. 1961) 465-470. 